\title{
Insulin Degradation. XXVIII. Immunocytochemical Localization of Glutathione-Insulin Transhydrogenase in the Pancreas, Kidney and Liver of Normal and Streptozotocin-Diabetic Rats and of Lean and Obese (ob/ob) Mice
}

\author{
C. A. Taylor and P. T. Varandani \\ Fels Research Institute and Department of Biological Chemistry, School of Medicine and College of Science and Engineering, \\ Wright State University, Yellow Springs, Ohio, USA
}

Summary. Glutathione-insulin transhydrogenase catalyzes the inactivation of insulin by splitting the hormone into $A$ and $B$ chains. We have localized this enzyme immunocytochemically by light microscopy in the pancreas, kidney and liver of both lean and obese (ob/ob) mice and similarly in normal and streptozotocin-diabetic rats. Localization was achieved by an antibody to glutathione-insulin transhydrogenase using a peroxidase-antiperoxidase technique. In comparison with tissues from control animals, positive immunostaining for glutathione-insulin transhydrogenase was increased in the obese mouse but reduced in the diabetic rat. Different tissues showed considerable variation in the amount of glutathioneinsulin transhydrogenase which could be detected. In the pancreatic islets there was little or no evidence for the presence of the enzyme in peripheral cells. In the kidney, immunocytochemical staining was found only in the proximal tubules. In the liver there was a generalised distribution of the enzyme, but the greatest concentration was in the periportal region. These observations parallel the biochemical data relating to glutathione-insulin transhydrogenase, indicating that different amounts of insulin degrading activity exist in different regions of tissues.

Key words: Immunocytochemistry, glutathione-insulin transhydrogenase, thiol:protein-disulphide oxidoreductase, streptozotocin-diabetic rats, obese (ob/ ob) mice, pancreatic acini, islets of Langerhans, kidney tubules, liver.

Glutathione-insulin transhydrogenase (GIT, thiol: protein disulphide oxidoreductase, E.C. 1.8.4.2) in vitro under appropriate conditions catalyzes, via sul- phydryl-disulphide interchange, the formation of correct disulphide bonds in scrambled forms of proteins such as ribonuclease, lysozyme and proinsulin. In addition, it cleaves disulphide bonds in native polypeptides such as insulin, proinsulin, oxytocin and vasopressin (with insulin as the most preferred substrate among the naturally occurring disulphide substrates) [ 25 for a recent review]. The cleavage of disulphide bonds of insulin by GIT results in the inactivation of the hormone and the formation of A and B chains [24]. This reaction, i. e. the splitting of the hormone by GIT, is the first step in the sequential pathway of insulin degradation, both at physiological and pharmacological concentrations of insulin $[19,21,29,34]$.

GIT is ubiquitous [2]. Work using several models (normal, starved, starved-refed, alloxan-diabetic, streptozotocin-diabetic rats; obese and lean mice) has shown that the concentration of liver enzyme is under feedback control by the circulating level of insulin [5, $16,17,18,23,30,32]$. Several factors (mono-, di and protein thiols [4] glucagon and growth hormone [31], adrenalectomy [11], glucocorticoids [5] and certain phospholipids [28]) have been found to modulate the activity of GIT [24 for a review]. In addition to regulation by these biochemical compounds, several different lines of evidence are suggestive that cell architecture probably plays an important role in the expression of the activity of the enzyme.

In the rat liver, the enzyme occurs bound to membranes as a phospholipoprotein complex in latent and nonlatent (active) forms ([20]. There is evidence that the proportions of the two forms are different under different pathological and physiological conditions $[16,30]$ and that the degree of latency may be related to the nature of the phospholipid composition of the membrane [26]. The enzyme contains multiple sites of reaction [28] and, as discussed previously [25], the in teractions in situ of different reaction sites with mem- 

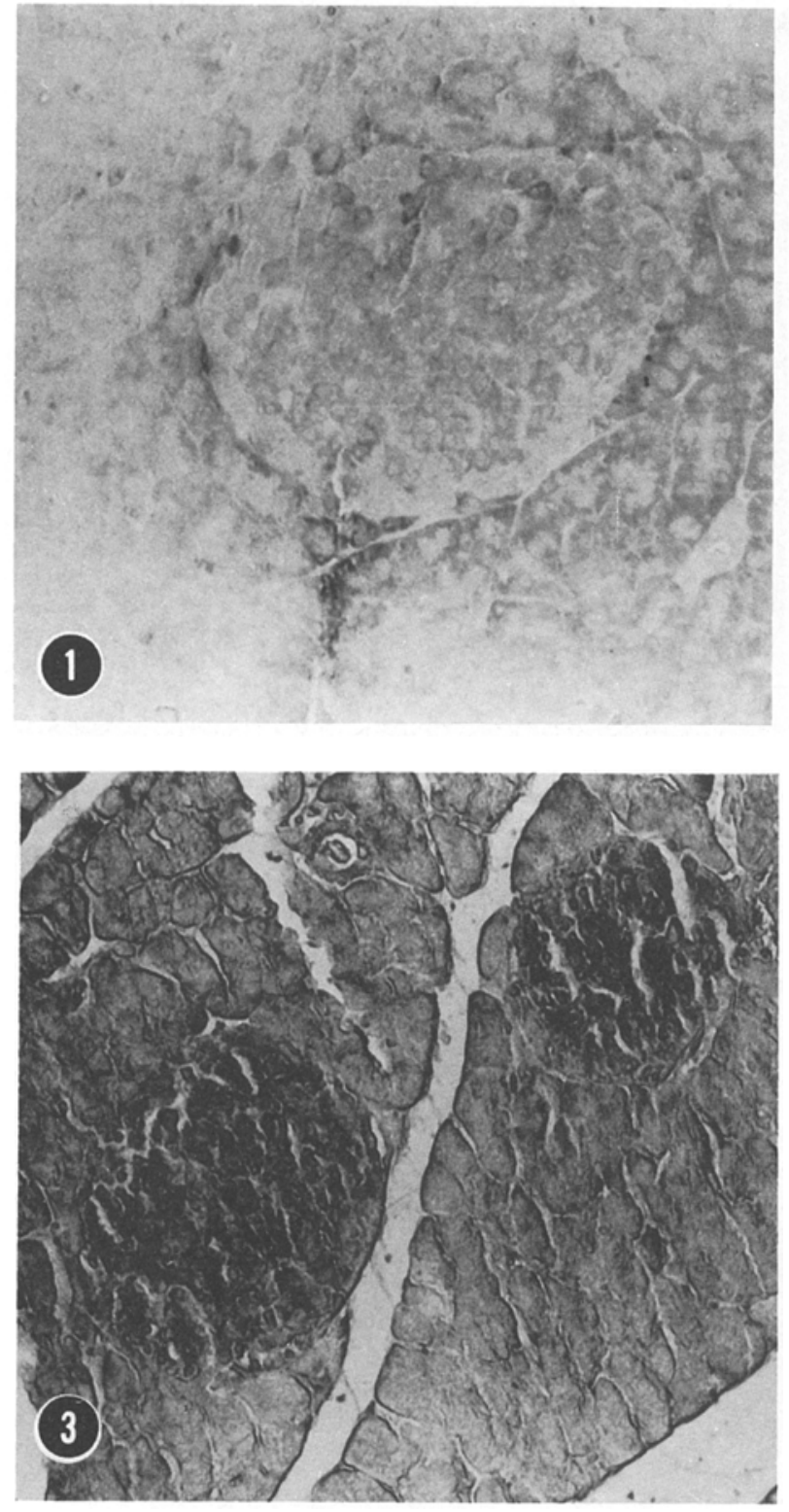

brane components (such as phospholipids [26, 28]) provide different compartments and micro-environments, thereby resulting in selectivity and different local effective enzyme activities.

Electron microscopy studies following immunoferritin labelling of unfixed isolated hepatocytes showed that GIT is located mainly on the inner side of the plasma membrane and intracellular membranes with relatively little GIT on the cell surface [33]. In the present paper, GIT was localized immunocytochemically using light microscopy. Fixed tissue sections were reacted with an antiserum against GIT and visualized by the peroxidase-antiperoxidase technique $[14,15]$. Using this method, we have studied the distribution of GIT in pancreas, kidney and liver ob-

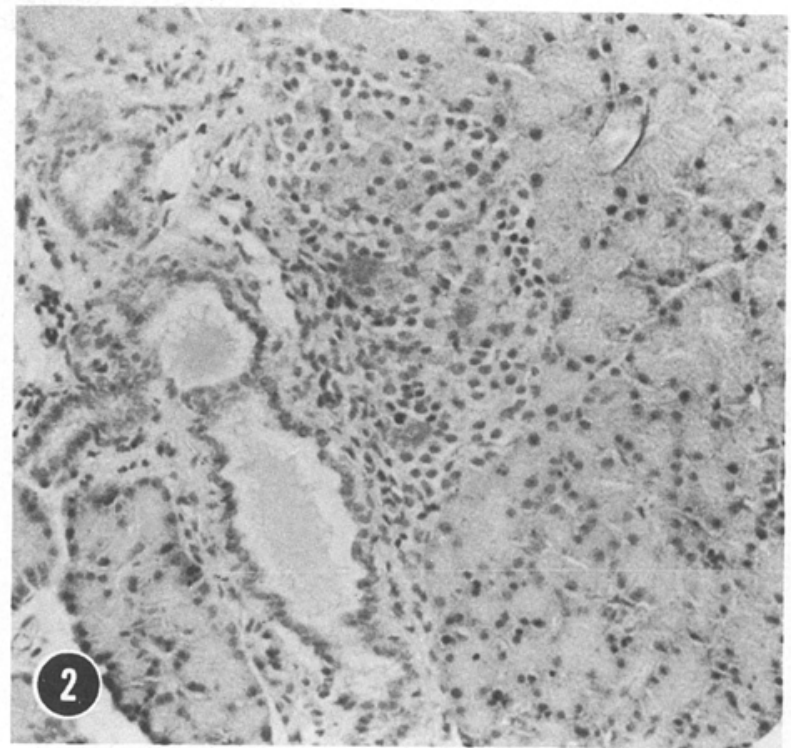

Fig. 1. Normal rat pancreas fixed in $4 \%$ paraformaldehyde- $0.5 \%$ glutaraldehyde. Section treated with GIT antiserum and peroxidase-antiperoxidase; this section was not counterstained. Both acinar and islet cells show immunoreactive product, although not all cells are equally reactive. Peripheral islet cells show little or no reactivity. $200 \times$

Fig. 2. Streptozotocin-diabetic rat pancreas. Section treated with GIT antiserum, peroxidase-antiperoxidase and counterstained with haematoxylin. Surviving islets appear disorganized : their outlines are irregular and there are spaces within the islets. OccasionalIy, nuclei surrounded by immunoreactive GIT product are found in the islet. $200 \times$

Fig. 3. Obese mouse pancreas. Section treated with GIT antiserum and peroxidase-antiperoxidase; this section was not counterstained. Compared with the lean mouse pancreas, GIT immunoreactivity is increased both in acinar cells and islets, although it is much more enhanced in the islets. $200 x$

tained from normal and streptozotocin-diabetic rats (as a model for hypoinsulinaemia) and from lean and obese (ob/ob) mice (as a model for hyperinsulinaemia).

\section{Materials and Methods}

\section{Materials}

Streptozotocin was supplied by Sigma, paraformaldehyde by Mallinckrodt, St. Louis, glutaraldehyde by Eastman Kodak, Rochester, New York, Tris by ICN K \& K Laboratories, Cleveland, Ohio, goat serum by Grand Island Biological Company, Grand Island, New York, anti-rabbit globulin made in goats and peroxidase-antiperoxidase complex made in rabbits by Litton Bionetics, Kensington, 


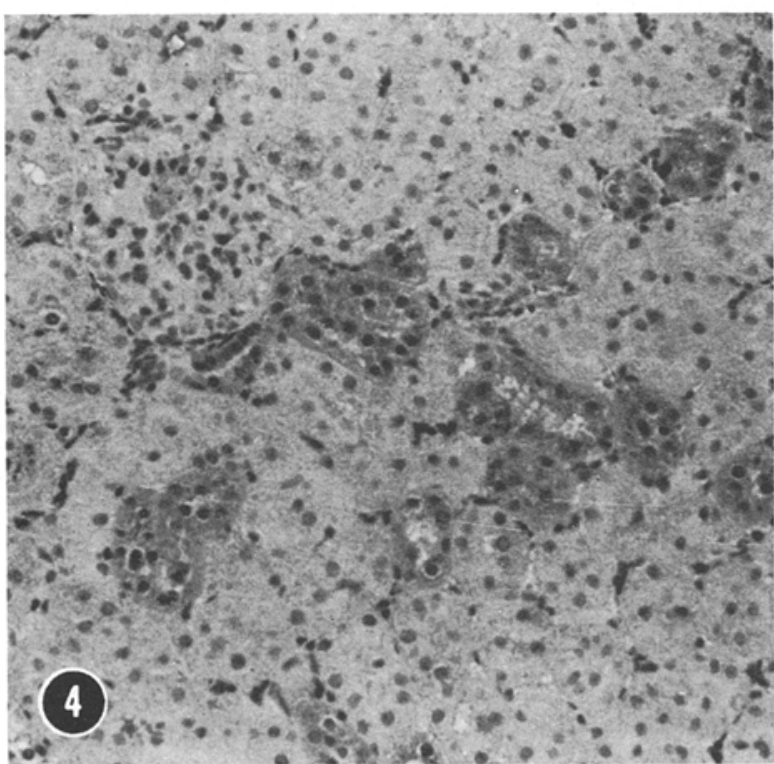

Fig. 4. Normal rat kidney cortex. Section treated with GIT antiserum, peroxidase-antiperoxidase and counterstained with haematoxylin. GIT immunoreactive product is concentrated in proximal tubules with different degrees of intensity; some tubules within the same region do not show any product. Glomeruli are unreactive. $200 \times$

Maryland. The soluble form (3, 4, 3', 4' -tetraamino-biphenyl hydrochloride) of $3,3^{\prime}$-diaminobenzidine (Sigma) was used [7]. The preparation and specificity of the antiserum to rat liver GIT were described previously [27]; immunoglobulin fraction precipitated by ammonium sulphate (33\%) was used throughout the studies. In Ouchterlony double-diffusion tests, the antibody shows a single precipitation band with purified GIT and tissue homogenates and no reaction with pork insulin, bovine albumin and bovine gamma globulin.

\section{Animals}

Adult male Sprague-Dawley rats (Harlan Industries, Indianapolis), weighing about $150 \mathrm{~g}$ each, were made diabetic by the IV injection of streptozotocin at a dose of $60 \mathrm{mg} / \mathrm{kg}$ body weight; control animals were injected with sodium citrate buffer. Details of our procedure have been previously described [16]. The diabetic animals had an elevated level of blood glucose $(>5 \mathrm{mg} / \mathrm{ml})$, a low circulating level of insulin ( $<5$ versus approximately $30 \mathrm{mU} / 1$ in normal animals) and survived for a prolonged period (at least 8 weeks) without exogenous insulin [16]. In the current studies the diabetic rats were sacrificed 3 weeks after the streptozotocin injection.

Obese-hyperglycaemic (ob/ob) $\mathrm{C} 57 \mathrm{BL} / 6 \mathrm{~J}$ male mice and their lean litter mates were purchased from Jackson Laboratories, Bar Harbor, Maine. Mice were 8 weeks old at the time of sacrifice, with body weights of $40.4-46.7 \mathrm{~g}$ for obese and $24.2-25.4 \mathrm{~g}$ for lean litter mates. Serum glucose levels of the obese mice were $4.0-6.8 \mathrm{mg} / \mathrm{ml}$ and those of the lean mice $1.1-1.8 \mathrm{mg} / \mathrm{ml}$.

\section{Tissue Preparation}

Animals were killed by decapitation. The pancreas, median lobe of the liver and cortex of the kidney were rapidly removed and placed

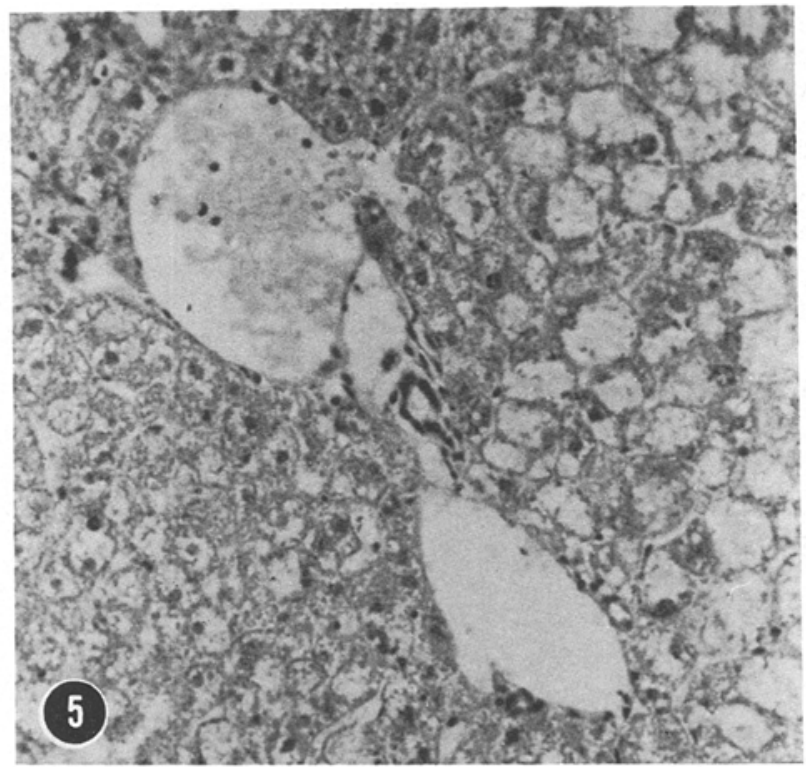

Fig. 5. Obese mouse liver. Section treated with GIT antiserum, peroxidase-antiperoxidase and counterstained with haematoxylin. Compared with lean mouse liver, obese mouse liver shows greatly increased immunoreactive GIT product which is concentrated in the periportal region surrounding the hepatic portal vein. Red blood cells within the vein also show a faint positive immunoreaction. Enlarged hepatic cells predominate in the obese-hyperglycaemic syndrome. $200 \times$

in $4 \%$ paraformaldehyde- $0.5 \%$ glutaraldehyde buffered at $\mathrm{pH} 7.5$ with $0.1 \mathrm{~mol} / 1$ potassium phosphate. Several $3 \mathrm{~mm}$ cubes were dissected from each tissue and transferred to fresh fixative. Three changes of fixative were made during the 30-60 min fixation period. For comparison, in some cases Bouin's solution was used as a fixative. Routine histological methods were used for dehydration in graded ethanols to dioxane and embedding in paraffin. Sections, $6 \mu \mathrm{m}$ thick, were mounted on acid-cleaned slides and dried overnight on a slide warmer. Several hundred sections from each of 50 different tissue blocks from a total of 13 animals (five obese and two lean mice; two diabetic and four normal rats) were studied.

\section{Peroxidase-Antiperoxidase Immunocytochemistry}

GIT was localized using the peroxidase-antiperoxidase techniques of Sternberger, et al. [14] and Taylor [15]. All procedures described below were carried out at room temperature. The mounted sections were deparaffinized in xylene and rehydrated with graded ethanols. Sera were diluted in $0.01 \mathrm{~mol} / 1$ Tris- $0.154 \mathrm{~mol} / 1$ saline $(\mathrm{pH}$ 7.5 ) containing $1 \%$ normal goat serum. Thirty microliters of each solution were used per section and slides were kept in a moist chamber during incubation. After each step, excess fluid was removed by draining and wiping around each section. Sections were subjected routinely to two treatments to minimize non-specific staining: blockage of endogenous peroxidase by treatment with $0.3 \% \mathrm{H}_{2} \mathrm{O}_{2}$ for $15 \mathrm{~min}$ followed by three $5 \mathrm{~min}$ washes with saline and application of normal goat serum $1: 10$ for $15 \mathrm{~min}$. Sections were then incubated sequentially for $30 \mathrm{~min}$ with the primary antiserum to GIT (1:100), a bridging antiserum (goat antibody against rabbit immunoglobulin diluted $1: 10$ ), and rabbit peroxidase-antiperoxidase complex $(1: 20)$. Each of these steps was followed by three 5 min washes in Tris-saline buffer containing goat serum. The washed sections were then exposed for $15 \mathrm{~min}$ to $0.05 \%$ diamino- 
benzidine followed by oxidation with $\mathrm{H}_{2} \mathrm{O}_{2}$ (final concentration of $0.01 \%$ ) for $5 \mathrm{~min}$ to form a brown reaction product. Nuclear counter-staining was carried out by immersing slides for $15 \mathrm{~s}$ in halfstrength Harris' haematoxylin. After rapid dehydration through graded ethanols and clearing overnight in xylene, sections were mounted in Depex.

Controls for non-specific staining included (a) substitution of normal rabbit serum far the primary antiserum, (b) use of primary antiserum previously absorbed against GIT [12], and (c) entry into the peroxidase-antiperoxidase sequence at each of the subsequent steps. The presence or absence of the brown immunoreactive product was verified independently by us and by three other investigators (two morphologists and one biochemist) who did not know the origin of tissue sections.

\section{Results}

Preliminary experiments using different fixatives showed that the fixation in $4 \%$ paraformaldehyde$0.5 \%$ glutaraldehyde provided adequate preservation of tissue morphology without destruction of GIT antigenicity. Figures directly pertinent to the overall conclusion are shown.

\section{Rat Pancreas}

Both the acinar cells and the islets of Langerhans in normal rat pancreas contain the diaminobenzidine immunoreactive GIT product (Fig. 1). A ring of less or non-reactive cells surrounds many islets. The immunoreactive product is absent in control sections.

The streptozotocin-injected rats were diabetic as indicated by glucosuria $(>5 \mathrm{mg} / \mathrm{ml})$. Three weeks after the streptozotocin injection most islets are unidentifiable. The few surviving islets appear disorganized (Fig. 2); their outlines are irregular and internal spaces or vacuities appear, apparently due to destruction of B cells. Acinar cells of diabetic rats show reduced intensity of GIT reactivity as compared with acinar cells of normal rats. Occasionally a few nuclei surrounded by immunoreactive GIT material are discernible among the spaces in the surviving islet (Fig. 2). Control sections show virtually no immunoreactive product.

\section{Mouse Pancreas}

As in the rat, acinar cells as well as islets show immunoreactive GIT product with the obese mouse containing more product than the lean mouse. Judging from the intensity of the reaction product, obese mouse islets contain much more GIT immunoreactive product than do acinar cells (Fig. 3). Examination of sections from different blocks of obese pancreas showed that different hypertrophied islets demonstrate different degrees of staining intensity. These differences do not seem to be due to artifactual entrapment of reactants under the sections because two islets within the same section show markedly different degrees of immunoreactive intensities but both islets are surrounded by less reactive acinar tissue. Furthermore, serial sections from the same tissue block exhibit the same variations from section to section. Adjacent sections treated with GIT antiserum previously absorbed against GIT or with non-immune serum are uniformly pale.

We also studied the localization of GIT in kidney and liver sections. Consistent with the findings of biochemical studies $[16,23,30]$, there seems to be a greater degree of immuno-intensity in tissues from hyperinsulinaemic models than in those from hypoinsulinaemic models. Interestingly, again both kidney and liver show regional localization of GIT. For example, in the normal rat kidney (Fig. 4) a striking pattern of immunoreactive GIT is localized in specific proximal convoluted tubules. Immunostaining occurs with differential intensity in typical proximal tubules from the renal cortex. Strands of immunoreactive material extend into the lumen. Glomeruli and many proximal tubules in the same vicinity are unreactive. Control sections treated with non-immune rabbit serum in lieu of the GIT antiserum are visible only when counterstained. Liver sections, e. g., from the obese mouse (Fig. 5), show GIT more concentrated in periportal hepatocytes. This concentration is most pronounced in the obese mouse and reflects the markedly increased GIT content in the obese tissue [30]. The liver cells from rats and lean mice show the usual hepatic morphology. In the obese liver (Fig. 5), cell boundaries which are immunoreactive stand out; the enlarged cells appear partially empty possibly because of the extraction during dehydration of the lipid material that is known to accumulate in the obese hyperglycaemic syndrome [8]. The faint immunostaining seen in red blood cells within the lumen of the hepatic portal vein can be correlated with the presence of GIT in the red blood cells [3]. No immunoreaction in the tissue or in the portal vein is observed when normal serum is substituted for primary antiserum.

\section{Discussion}

The development of an immunocytochemical procedure for the localization of GIT in fixed tissues using light microscopy allows the identification of GIT within tissue regions and individual cells. Such data may add significant information about cell function. The distribution of GIT warrants further examination with the electron microscope in order to pinpoint the subcellular localization of the enzyme and to study 
the effects of the diabetic and obese states on cell ultrastructure.

The results of our immunocytochemical studies of pancreatic tissue show that both acini and islets contain GIT. While our work was in progress, localization of GIT in islets and acinar cells using frozen sections of normal rat pancreas was reported [6]. Several lines of evidence indicate that in the islets of Langerhans, GIT is preferentially associated with B cells. (a) The GIT immunostaining is less intense in the peripheral cells where most A cells (which contain glucagon) and D cells (which contain somatostatin) occur [1]. (b) Streptozotocin which selectively causes destruction of B cells also results in the loss of GIT immunostaining in the islets. (c) The ob/ob obese (C57BL/6J) syndrome in which the B cell number is known to increase [1] also shows a marked increase in GIT immunostaining. Although there are no biochemical data on GIT in the pancreatic islets, the findings in the present studies indicate that there are increased amounts of GIT in the islets of obese mice. This supports the suggestion [22] that the function of GIT in the pancreatic islets is to promote the biosynthesis of proinsulin and insulin rather than their degradation, resulting in an increased circulating level of insulin in the ob/ob mice. This interpretation is consistent with the finding of an occasional islet (with minimal GIT reactivity) in streptozotocin diabetic rats.

The present immunocytochemical data clearly indicate that the GIT-positive immunostaining is greatly increased in obese mice (characterized by hyperinsulinaemia) but is reduced in diabetic rats (characterized by hypoinsulinaemia) compared with the control animals. However, the same cell types do not necessarily contain the same amounts of GIT. In the pancreas of obese mice the intensity of immunoreactivity varies from one islet to another within the same section or within the same tissue block, suggesting that all islets do not have an equal amount of GIT activity. In the kidney, there are clusters of GIT-rich proximal tubules; but, in the same region other proximal tubules are unreactive. In the liver, GIT is concentrated in the periportal region which is emphasized in obese mice. Consistent with the immunocytochemical data, biochemical assays on liver homogenates from obese mice have shown elevated levels of GIT [30] and from diabetic rats have shown reduced levels of GIT [16, $17,23]$. However, studies using isolated perfused intact livers have shown the opposite results $[9,10,13]$, i. e. elevated insulin clearance by diabetic livers and depressed clearance by obese livers. These apparent discrepancies in the biochemical data can now be reconciled on the basis of information provided by the current studies. Studies using liver homogenates yield average values whereas studies using isolated perfused intact liver indicate the function of a specific hepatic region [5], since the occurrence of GIT-rich and GIT-poor regions in tissues probably results in different net effective activities in different cellular compartments or local regions. Thus, while circulating insulin may represent a major control mechanism over GIT concentration, the occurrence of variable local concentrations of GIT might provide, as previously proposed [25], a mechanism by which the enzyme regulates the cleavage and/or the formation of disulphide bonds in proteins, depending upon the substrate [31], tissue and/or metabolic state of the cell. However, further work will be necessary to examine these possibilities.

Acknowledgement. This work was supported in part by USPHS Grant AM-24057.

\section{References}

1. Beatens D, Stefan Y, Ravazzola M, Malaisse-Lagae F, Coleman DL, Orci L (1978) Alteration of islet cell populations in spontaneously diabetic mice. Diabetes $27: 1-7$

2. Chandler ML, Varandani PT (1972) Insulin degradation. II. The wide-spread distribution of glutathione-insulin transhydrogenase in the tissues of the rat. Biochim Biophys Acta 286: 136-145

3. Chandler ML, Varandani PT (1974) Insulin degradation. IX. On the presence of glutathione-insulin transhydrogenase in human leukocytes. Diabetes 23:232-239

4. Chandler ML, Varandani PT (1975) Kinetic analysis of the mechanism of insulin degradation by glutathione-insulin transhydrogenase (thiol:protein disulfide oxidoreductase). Biochemistry $14: 2107-2115$

5. Cudworth AG, Barber HE (1975) The effect of hydrocortisone phosphate, methylpredinisolone and phenytoin on pancreatic insulin release and hepatic glutathione-insulin transhydrogenase activity in the rat. Eur J Pharmacol $31: 23-38$

6. Dorn A, Kohnert K-D, Ansorge S, Zühlke H. Arlt B, Lorenz D (1978) The immunohistochemical demonstration of the thiol: protein disulfide oxidoreductase (TPO) in pancreas and isolated Langerhans' islets: a light, fluorescent and electron microscopic study. Acta Histochem 63:200-208

7. Graham RC, Karnovsky MJ (1966) The early stages of absorption of injected horseradish peroxidase in the proximal tubules of mouse kidney: ultrastructural cytochemistry by a new technique. J Histochem Cytochem 14: 291-301

8. Herzberg L, Coleman DL (1977) Laboratory animals exhibiting obesity and diabetes syndromes. Metabolism 26:59-99

9. Karakash C, Assimacopoulos-Jeannet F, Jeanrenaud B (1976) An anomaly of insulin removal in perfused livers of obese hyperglycemic (ob/ob) mice. J Clin Invest 57: 1117-1124

10. McCarroll AM, Buchanan KD (1973) Insulin clearance by the isolated perfused livers of insulin deficient rats. Diabetologia 9: 457-460

11. Petri W Jr, Rodriguez J, Pitot HC (1976) Environmental effects on glutathione-insulin transhydrogenase in rat liver. Proc Soc Exp Biol Med 152: 610-614

12. Phelps BH, Varandani PT (1977) Stimulation by insulin of cyclic AMP phosphodiesterase. Role of glutathione-insulin transhydrogenase. Biochem Biophys Res Commun 75:302-310 
13. Sacks H, Pimstone B, Waligora K, Peires L, Weinkove E, Saunders S (1977) Differences between insulin degradation by soluble fractions and insulin clearance by perfused livers of normal, protein-depleted, and starved rats. Diabetes 26:956-966

14. Sternberger LA, Hardy PH, Cuculis JJ, Meyer HG (1970) The unlabeled antibody enzyme method of immunohistochemistry. Preparation and properties of soluble antigen-antibody compley (horseradish peroxidase-antihorseradish peroxidase) and its use in identification of spirochetes. J Histochem Cytochem 18:315-333

15. Taylor CR (1978) Immunocytochemical methods in the study of lymphoma and related conditions. J Histochem Cytochem $26: 496-512$

16. Thomas JH, Varandani PT (1979) Insulin degradation. XXV. Glutathione-insulin transhydrogenase activity of rat liver and kidney during the development of streptozotocin-diabetes. Biochim Biophys Acta 567: 88-95

17. Thomas JH, Wakefield SM, Jones RH (1973) The effect of insulin deficiency on the glutathione-insulin transhydrogenase activity of rat liver. Biochem Soc. Trans 1: 1179-1182

18. Uete T, Shimano N, Shimizu S, Morikawa M (1976) Autoregulatory system of insulin degradation in liver. II. Relationship between blood insulin levels and GSH-dependent insulin degrading activity in liver and blood. Metabolism $25: 375-384$

19. Varandani PT (1973) Insulin degradation. IV. Sequential degradation of insulin by rat kidney, heart and skeletal muscle homogenates. Biochim Biophys Acta 295: 630-636

20. Varandani PT (1973) Insulin degradation. V. Unmasking of glur tathione-insulin transhydrogenase in rat liver microsomal membrane. Biochim Biophys Acta 304: 642-659

21. Varandani PT (1973) Insulin degradation. VII. Sequential degradation of insulin by rat liver homogenates at physiological concentrations of insulin and in the absence of exogenous glutathione. Biochim Biophys Acta $320: 249-257$

22. Varandani PT (1974) Insulin degradation in insulinoma: evidence for the occurrence of an inactive form of glutathione-insulin transhydrogenase and for the absence of insulin A and B chains degrading protease(s). Biochem Biophys Res Commun 60: $1119-1126$

23. Varandani PT (1974) Insulin degradation. VI. Feedback control by insulin of liver glutathione-insulin transhydrogenase in rat. Diabetes 23: 117-125

24. Varandani PT (1976) The role of glutathione-insulin transhydrogenase in insulin degradation. In: Proc. IX Congress of the International Diabetes Federation (New Delhi, India). Excerpta Medica, Amsterdam, pp 213-223
25. Varandani PT (1978) Mechanistic and structural aspects of glutathione-insulin transhydrogenase (protein-disulfide interchange enzyme). In: Singer TP, Ondarza RN (eds) Mechanisms of oxidizing enzymes. Elsevier/North-Holland, New York, pp $29-42$

26. Varandani PT, Darrow RM, Nafz MA (1977) Phospholipid composition of liver homogenates and microsomes of the lean and obese hyperglycemic (ob/ob) mouse. Proc Soc Exp Biol Med 156: 123-126

27. Varandani PT, Nafz MA (1972) Insulin degradation. I. Purification and properties of glutathione-insulin transhydrogenase of rat liver. Biochim Biophys Acta 286: 126-135

28. Varandani PT, Nafz MA (1976) Interaction of glutathione-insulin transhydrogenase (disulfide interchange enzyme) with phospholipids. Biochim Biophys Acta 438: 358-369

29. Varandani PT, Nafz MA (1976) Insulin degradation. XVI. Evidence for the sequential degradative pathway in isolated liver cells. Diabetes 25: 173-179

30. Varandani PT, Nafz MA (1976) Insulin degradation. XVIII. On the regulation of glutathione-insulin transhydrogenase in the hyperglycemic obese (ob/ob) mouse. Biochim Biophys Acta $451: 382-392$

31. Varandani PT, Nafz MA, Chandler ML (1975) Interaction of insulin analogs, glucagon, growth hormone, vasopressin, oxytocin, and scrambled forms of ribonuclease and lysozyme with glutathione-insulin transhydrogenase (thiol: protein disulfide oxidoreductase): dependence upon conformation. Biochemstry $14: 2115-2120$

32. Varandani PT, Nafz MA, Shroyer LA (1971) Glutathione-insulin transhydrogenase: evidence for a key role in insulin metabolism. Diabetes 20 [Supp1 1]: 342

33. Varandani PT, Raveed D, Nafz MA (1978) Insulin degradation XXIII. Distribution of glutathione-insulin transhydrogenase in isolated rat hepatocytes as studied by immuno-ferritin and electron microscopy. Biochim Biophys Acta 538: 343-353

34. Varandani PT, Shroyer LA, Nafz MA (1972) Sequential degradation of insulin by rat liver homogenates. Proc Natl Acad Sci USA $69: 1681-1684$

Received: 30 December 1980

and in revised form: 27 February 1981

Dr. P. T. Varandani

Fels Research Institute

800 Livermore Street

Yellow Springs, OH 45387, USA 